\title{
MAGNETIC FIELDS AND INFALL MOTIONS IN NGC 1333 IRAS 4
}

\author{
Michael Attard ${ }^{1}$, Martin Houde $^{1}$, Giles NovaK ${ }^{2},{\text { Hua-bai } \text { Li }^{3} \text {, John E. Vaillancourt }}^{4}$, C. Darren Dowell ${ }^{4,5}$, \\ JACQUELINE DAVIDSON $^{6}$, AND HiROKO SHINNAGA ${ }^{7}$ \\ ${ }^{1}$ Department of Physics and Astronomy, The University of Western Ontario, London, ON N6A 3K7, Canada; mattard@uwo.ca \\ ${ }^{2}$ Department of Physics and Astronomy, Northwestern University, Evanston, IL 60208, USA \\ ${ }^{3}$ Harvard-Smithsonian Center for Astrophysics, MS-78, Cambridge, MA 02138, USA \\ ${ }^{4}$ Division of Physics, Mathematics, \& Astronomy, California Institute of Technology, 1200 E. California Blvd., MS 320-47, Pasadena, CA 91125, USA \\ ${ }^{5}$ Jet Propulsion Laboratory, California Institute of Technology, Pasadena, CA 91109, USA \\ ${ }^{6}$ School of Physics, The University of Western Australia, Crawley, WA 6009, Australia \\ ${ }^{7}$ Caltech Submillimeter Observatory, 111 Nowelo St., Hilo, HI 96720, USA \\ Received 2009 May 4; accepted 2009 July 8; published 2009 August 24
}

\begin{abstract}
We present single-dish $350 \mu \mathrm{m}$ dust continuum polarimetry as well as $\mathrm{HCN}$ and $\mathrm{HCO}^{+} J=4 \rightarrow 3$ rotational emission spectra obtained on NGC 1333 IRAS 4 . The polarimetry indicates a uniform field morphology over a $20^{\prime \prime}$ radius from the peak continuum flux of IRAS 4A, in agreement with models of magnetically supported cloud collapse. The field morphology around IRAS 4B appears to be quite distinct, however, with indications of depolarization observed toward the peak flux of this source. Inverse P Cygni profiles are observed in the $\mathrm{HCN} J=4 \rightarrow 3$ line spectra toward IRAS 4A, providing a clear indication of infall gas motions. Taken together, the evidence gathered here appears to support the scenario that IRAS 4A is a cloud core in a critical state of support against gravitational collapse.

Key words: ISM: individual (NGC 1333 IRAS 4) - ISM: magnetic fields - ISM: molecules - polarization - stars: formation - submillimeter
\end{abstract}

Online-only material: color figures

\section{INTRODUCTION}

The process of star formation is a key phenomenon in astrophysics. It touches upon a diverse range of topics including galactic evolution, stellar evolution, planet formation, and astrobiology. Yet despite the fundamental nature of this process, it remains poorly understood (Crutcher et al. 2009). A key issue is determining the support mechanism(s) that governs star formation, as it is clear that stars cannot be formed at a free fall timescale (Shu et al. 1987). Two competing ideas currently strive to explain the support mechanism: magnetism plus ambipolar diffusion and turbulence plus a weak magnetic field (McKee $\&$ Ostriker 2007). At present, a consensus on the nature of the support mechanism has not been realized.

A particularly important quantity for star formation theory is the mass-to-flux ratio, $M / \Phi$, where $\Phi$ is the magnetic flux within a region enclosing a mass $M$ (Nakamura \& Li 2008). Magnetic support models predict the critical value of this ratio to be equal to $c_{\Phi} / \sqrt{G}$ (Mouschovias \& Spitzer 1976), where $G$ is the gravitational constant and $c_{\Phi}=0.12$ (Tomisaka et al. 1988). It follows that the value of $M / \Phi$ normalized to $c_{\Phi} / \sqrt{G}$ should be close to unity in regions near the point of collapse. Turbulent models place no such restrictions on the mass-to-flux ratio but do predict a chaotic field morphology assuming that flux-freezing holds. The mass-to-flux ratio can be determined from observables and thus may be used as an important indicator as to the nature of the support mechanism.

At a distance of $\approx 300^{8}$ (Girart et al. 2006), a known site of clustered low and intermediate mass star formation, and possessing young embedded cores at an age of $\approx 1 \mathrm{Myr}$ (Hatchell et al. 2005), NGC 1333 is an ideal target for studying the onset of clustered star formation. Two such embedded cores of interest include NGC 1333 IRAS 4A $\left(\alpha_{2000}=3^{\mathrm{h}} 29^{\mathrm{m}} 10.42^{\mathrm{s}}\right.$,

\footnotetext{
8 The distance of $300 \mathrm{pc}$ will be assumed throughout this work.
}

$\delta_{2000}=+31^{\circ} 13^{\prime} 35^{\prime \prime} \cdot 4$, henceforth 4A) and NGC 1333 IRAS 4B $\left(\alpha_{2000}=3^{\mathrm{h}} 29^{\mathrm{m}} 12.06^{\mathrm{s}}, \delta_{2000}=+31^{\circ} 13^{\prime} 10^{\prime \prime} 8\right.$, henceforth 4B $)$. Note that $\alpha_{2000}$ and $\delta_{2000}$ will denote J2000 epoch right ascension and declination coordinates throughout this paper, respectively ( $\alpha_{o}$ and $\delta_{o}$ will denote offsets in right ascension and declination from a particular reference point, respectively). The source 4A has been extensively studied in the past (Sandell et al. 1991; Di Francesco et al. 2001; Girart et al. 2006). We have carried out continuum polarization and spectroscopic observations using, respectively, SHARP at $350 \mu \mathrm{m}$ toward $4 \mathrm{~A} / 4 \mathrm{~B}$ and the 300-400 GHz heterodyne receiver at the Caltech Submillimeter Observatory (CSO) on 4A. It is these data and the subsequent analysis that are presented.

Previous work has greatly improved our knowledge of both $4 \mathrm{~A}$ and $4 \mathrm{~B}$. The interferometric detection of inverse P Cygni profiles toward both of these sources provides strong evidence for infalling gas motions (Di Francesco et al. 2001, hereafter DF2001). The detections were made in $\mathrm{H}_{2} \mathrm{CO} 3_{21} \rightarrow 2_{11}$ emission and have allowed the determination of infall speeds of 0.68 and $0.47 \mathrm{~km} \mathrm{~s}^{-1}$ for $4 \mathrm{~A}$ and $4 \mathrm{~B}$, respectively. DF2001 also provide simple mass estimates for the gas in each source and find $0.71 M_{\odot}$ and $0.23 M_{\odot}$ within corresponding radii of $9^{\prime \prime}(0.013)$ and $6^{\prime \prime}(0.009)$ from the peak flux of $4 \mathrm{~A}$ and $4 \mathrm{~B}$, respectively. Finally, mass accretion rates of $1.1 \times 10^{-4} M_{\odot} \mathrm{yr}^{-1}$ and $3.7 \times 10^{-5} M_{\odot} \mathrm{yr}^{-1}$ were calculated for both $4 \mathrm{~A}$ and $4 \mathrm{~B}$, respectively.

Recent $877 \mu \mathrm{m}$ continuum polarimetry done at the Submillimeter Array (SMA) has indicated the presence of a welldefined pinch in the magnetic field morphology around 4A (Girart et al. 2006, hereafter G2006). From these measurements the authors estimate the mass-to-flux ratio to be $\approx 1.7$ times the critical value of collapse. Along with their computation of the ratio of the turbulent to magnetic energy, $\beta_{\text {turb }} \equiv \sigma_{\text {turb }}^{2} / V_{A}^{2} \approx 8 \times 10^{-4}\left(\delta \theta_{\text {int }} / 1^{\circ}\right)^{2}=0.02$, where $\sigma_{\text {turb }}$ 
is the turbulent line width, $V_{A}$ is the Alfvén speed, and $\delta \theta_{\text {int }}$ is the intrinsic dispersion in the polarization vectors (Lai et al. 2002), the authors conclude that $4 \mathrm{~A}$ is an example of a magnetically dominated collapsing cloud core. A cloud mass of $1.2 M_{\odot}$ within a radial distance of $3^{\prime \prime}(0.004)$ from the peak flux of $4 \mathrm{~A}$ is also traced by their dust continuum measurements. Taken together, the DF2001 \& G2006 results present compelling evidence for the notion that the physics of $4 \mathrm{~A}$ is consistent with standard magnetically regulated star formation theory.

One problem not addressed in these works is the variation of physical parameters as a function of spatial scales. Of particular interest here is the variation in the magnetic field morphology with spatial scales; models predict that the regions undergoing collapse will drag in the field lines toward the central condensation producing an hourglass morphology. Further out from the condensation, the field morphology should remain in its ambient state (Fielder \& Mouschovias 1993). The results presented in G2006 illustrate an example of this hourglass morphology at a resolution of $\approx 1^{\prime \prime}(0.001)$. However, due to this small spatial scale G2006 was unable to sample the field morphology at scales larger than $\approx 10^{\prime \prime}(0.015)$ where models predict the field to be uniform. Similarly for the spectroscopy work, the high resolution attained through interferometry by DF2001 $(\approx 2$ " or 0.003$)$ allowed them to identify infall signatures out to a distance of $\approx 4^{\prime \prime}(0.006)$ from the peak flux of $4 \mathrm{~A}$ and $4 \mathrm{~B}$. It is not clear from their results whether or not infall motions are occurring further out from the peak positions.

The aim of this study is to address the problem of spatial scale variation in magnetic fields and infall motions and to complement the work of G2001 and DF2001. This will be done by analyzing single-dish observations obtained at larger spatial scales and comparing these results with the aforementioned papers. In the following sections, we describe both polarimetric and spectroscopic observations carried out at the CSO and discuss the implications of our findings. In Section 2, we discuss our observations. Section 3 will cover a general discussion and analysis of our results, and finally in Section 4 we state our conclusions.

\section{OBSERVATIONS}

The following two subsections describe the submillimeter dust continuum polarimetry and spectroscopic observations acquired by our group. In Section 2.1, we discuss $350 \mu \mathrm{m}$ polarimetry data collected in 2008 September using SHARP, the SHARC-II polarimeter. In Section 2.2, we will discuss the HCN $J=4 \rightarrow 3$ spectroscopic observations taken with the CSO 300-400 GHz heterodyne receiver in 2000 September.

\subsection{Polarimetry}

Dust continuum polarimetry was done with SHARP at $350 \mu \mathrm{m}$ with a spatial resolution of $\approx 10^{\prime \prime}(0.015)$. SHARP is a fore-optics addition to the SHARC-II camera that enables this instrument to be used as a sensitive polarimeter $(\mathrm{Li}$ et al. 2008). Although both $4 \mathrm{~A}$ and $4 \mathrm{~B}$ are studied here, the telescope was pointed onto $4 \mathrm{~B}$ so as to provide the best possible chance of detecting polarization on this fainter source. Our map was calibrated with approximately $3 \mathrm{hr}$ worth of data obtained on W3 $(\mathrm{OH})$ during the same observing run. In order to account for any random or systematic uncertainties that may remain after applying our standard data reduction pipeline, a reduced $-\chi^{2}$ analysis was performed on the data. The measurement uncer-
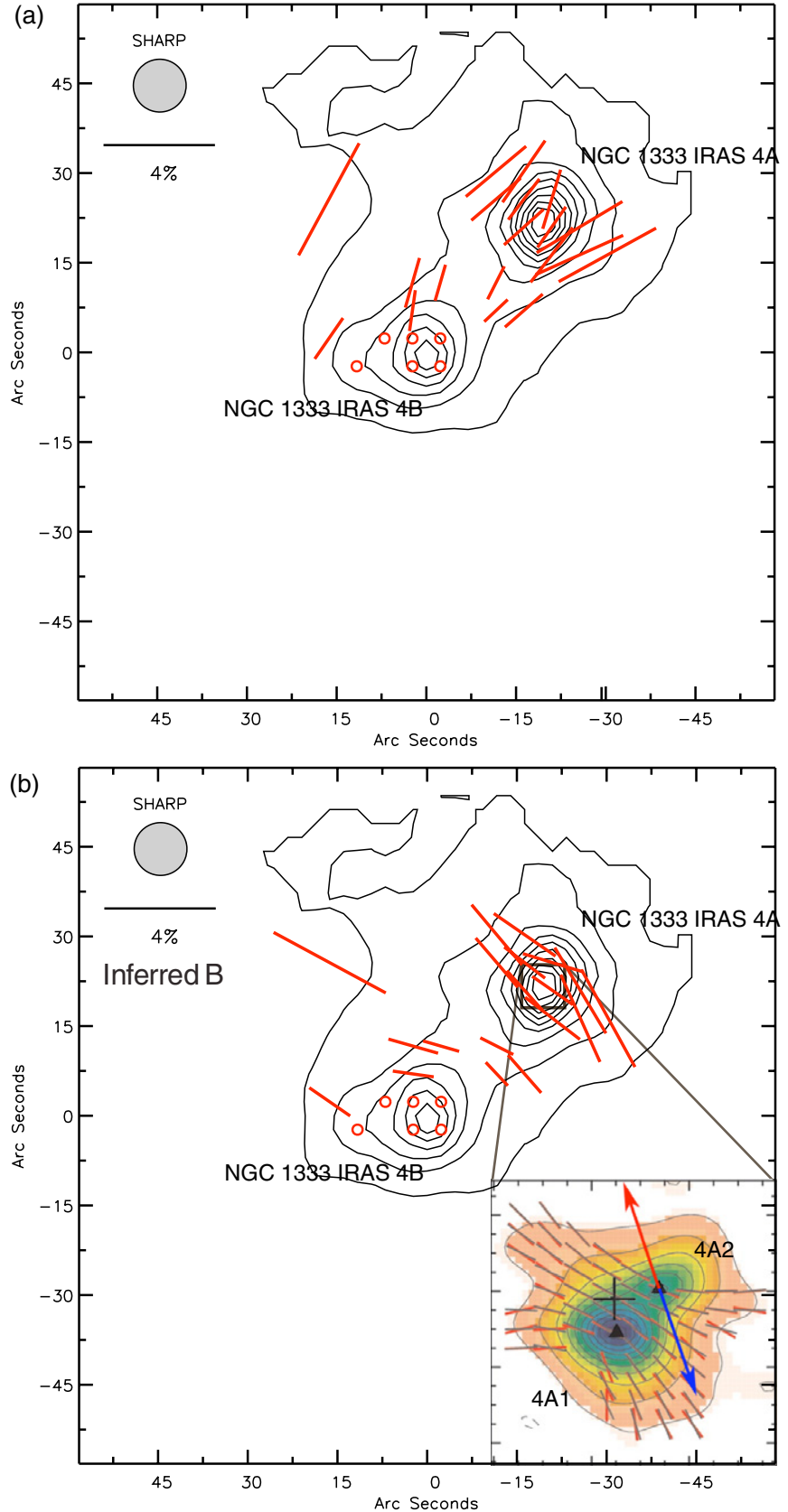

Figure 1. SHARP polarimetry (a) and deduced magnetic field orientation (b) over $4 \mathrm{~A}$ and $4 \mathrm{~B}$. Both images are centered on $4 \mathrm{~B}\left(\alpha_{2000}=3^{\mathrm{h}} 29^{\mathrm{m}} 12.06^{\mathrm{s}}\right.$, $\delta_{2000}=+31^{\circ} 13^{\prime} 10^{\prime \prime} 8$ ) with $4 \mathrm{~A}$ lying toward the northwest corner of the map. The horizontal and vertical axes show offsets in R.A. and decl., respectively. Contour levels are $0.1,0.2, \ldots, 0.9$ times the peak flux value $\left(29.3 \mathrm{Jy}\right.$ beam $\left.{ }^{-1}\right)$. Image (b) also shows the G2006 magnetic field map for comparison, where 4A is resolved into its components $4 \mathrm{~A} 1$ and $4 \mathrm{~A} 2$. The arrows indicate the orientation of the outflow. Note that all the vectors presented in image (a) are $p>3 \sigma_{p}$, and the circles denote regions where $p+2 \sigma_{p}<1 \%$.

(A color version of this figure is available in the online journal.)

tainty was correspondingly inflated on a pixel-by-pixel basis, such that the reduced $\chi^{2}=1$. Our results are shown in Figure 1 and represent approximately $10.5 \mathrm{hr}$ of observation time.

Several points as follows are worth mentioning from Figure 1. (1) The extended magnetic field around 4A is clearly sampled in our map. The nature of the extended field appears to be uniform out to a distance of $\approx 20^{\prime \prime}(0.03)$ from the peak of this source. This is consistent with the $850 \mu \mathrm{m}$, effective $20^{\prime \prime}$ beamwidth, SCUPOL observations taken at the James Clerk 
Maxwell Telescope (JCMT; Matthews et al. 2009). Our data enable a rough upper limit to be placed on the size of the magnetic pinch reported in G2006 to one SHARP resolution element ( $\sim 10^{\prime \prime}$ or 0.015$)$. (2) Deviations in the field appear as one moves out beyond $20^{\prime \prime}(0.03)$ from $4 \mathrm{~A}$ toward 4B. In the vicinity of $4 \mathrm{~B}$, the field morphology is rotated by $\sim 30^{\circ}$ toward the horizontal with respect to the field orientation around $4 \mathrm{~A}$. (3) Depolarization is observed toward the peak of $4 \mathrm{~B}$ as denoted by the open circles on the map where $p+2 \sigma_{p}<1 \%$. Here $p$ is the degree of polarization, and $\sigma_{p}$ is the corresponding uncertainty in the value of $p$. Significant depolarization is not observed toward $4 \mathrm{~A}$.

Taken together, these results suggest that $4 \mathrm{~A}$ and $4 \mathrm{~B}$ are magnetically distinct objects. While at first glance the polarimetry of $4 \mathrm{~A}$ appears to be consistent with conventional ideas of magnetic support, $4 \mathrm{~B}$ is a more complicated case. The observed depolarization on 4B may result from changes in the dust grain properties or shapes due to grain-growth (Vrba et al. 1993; Hildebrand et al. 1999), supersonic and super-Alfvénic turbulence plus a lack of grain alignment above $A_{V} \approx 3$ mag (Padoan et al. 2001), magnetic field geometry and the inclination angle with the line of sight (Goncalves et al. 2005; Fiege \& Pudritz 2000), or finally because of beam smearing over small-scale field structures (Rao et al. 1998). The last point could be tested in a straightforward manner with high-resolution polarimetry. In addition, Choi (2001) identifies a Class I object (denoted 4BII in the paper) within close proximity to the younger $4 \mathrm{~B}$ core. The existence of this more evolved object is linked to the "Cav2" cavity, located at the west of the $4 \mathrm{~B}$ complex, and may have generated this feature through the action of an ancient outflow (Choi 2001). It is possible to speculate that turbulence driven by this ancient outflow activity from 4BII may have disrupted the embedded magnetic field and thus contributed to the observed depolarization. We should note that no such Class I objects have been identified within the 4A complex (Choi 2005).

We now look at the position angles of the vectors situated within $20^{\prime \prime}$ of the peak of $4 \mathrm{~A}$ in order to assess the orientation of the large-scale magnetic field around this source. We assume that this large-scale field has a simple, uniform morphology with a polarization angle $\theta$. The value of $\theta$ can be calculated by computing the mean of the individual orientation angles $\theta_{i}$, where the subscript $i$ denotes the individual vectors. Table 1 contains all the relevant information on all the vectors depicted in Figure 1.

From the data presented in Table 1, a straight arithmetic average of the orientation angles $\theta_{i}$ for the vectors situated within a $20^{\prime \prime}$ radius of the peak of $4 \mathrm{~A}$ yields a mean value of $\theta \approx 45.9$. The dispersion in the orientation angles for these vectors $\left(\delta \theta_{\text {obs }}\right)$ is found to be $\approx \pm 13.6$. We therefore adopt $\theta \approx 45.9$ as the orientation angle of the large-scale uniform magnetic field around $4 \mathrm{~A}$. The G2006 result of $\approx 61^{\circ}$ for their field orientation is approximately a $1 \sigma$ deviation from our result, implying that the two values are consistent with one another. Uncertainties in our methods could account for the $\approx 15^{\circ}$ discrepancy; we admittedly assume a very simple uniform model for the field orientation, ignoring any possible non-uniform large-scale structure to the morphology. The main outflow from $4 \mathrm{~A}$ extends $\pm 2^{\prime}$ with a position angle of about $45^{\circ}$ for a line drawn from one tip of the outflow to the other, but this rotates to $19^{\circ}$ for the inner part of the outflow confined to a radius of approximately 40" (Blake et al. 1995; Choi 2005). The reason for the change in the orientation of the outflow is unknown; cloud core rotation may have played a role. A
Table 1

Measured Polarization for NGC 1333 IRAS $4^{\mathrm{c}}$

\begin{tabular}{cccccc}
\hline \hline $\begin{array}{l}\alpha_{o} \text { Offset }^{\mathrm{b}} \\
(\operatorname{arcsec})\end{array}$ & $\begin{array}{c}\delta_{o} \text { Offset }^{\mathrm{b}} \\
(\operatorname{arcsec})\end{array}$ & $\begin{array}{c}p \\
(\%)\end{array}$ & $\begin{array}{c}\sigma_{p} \\
(\%)\end{array}$ & $\begin{array}{c}\theta_{i}{ }^{\mathrm{a}} \\
\left({ }^{\circ}\right)\end{array}$ & $\begin{array}{r}\sigma_{\theta_{i}} \\
\left({ }^{\circ}\right)\end{array}$ \\
\hline 19.4 & 0.3 & 1.8 & 0.5 & 55.3 & 7.1 \\
13.9 & 21.1 & 4.6 & 1.2 & 61.6 & 8.8 \\
6.1 & 5.0 & 1.5 & 0.5 & 81.9 & 7.7 \\
5.0 & 9.5 & 1.9 & 0.5 & 74.1 & 8.0 \\
1.6 & 10.1 & 1.3 & 0.3 & 73.9 & 6.9 \\
\hline-8.1 & & & & & \\
-7.9 & 6.3 & 1.2 & 0.3 & 43.3 & 15.0 \\
-9.8 & 10.3 & 1.3 & 0.4 & 63.1 & 9.1 \\
-10.5 & 23.3 & 2.3 & 0.8 & 40.5 & 9.8 \\
-13.8 & 27.0 & 2.8 & 0.9 & 39.9 & 5.0 \\
-14.0 & 5.2 & 1.8 & 0.6 & 41.2 & 10.6 \\
-13.7 & 19.3 & 1.9 & 0.3 & 42.0 & 3.5 \\
-15.5 & 24.0 & 1.8 & 0.4 & 52.6 & 8.5 \\
-19.7 & 27.5 & 2.7 & 0.7 & 55.4 & 5.7 \\
-18.6 & 13.6 & 2.5 & 0.7 & 52.4 & 6.0 \\
-19.3 & 19.2 & 1.8 & 0.4 & 55.1 & 7.4 \\
-25.0 & 23.6 & 2.2 & 0.6 & 73.1 & 6.3 \\
-25.5 & 11.4 & 3.4 & 0.9 & 24.2 & 7.0 \\
-30.5 & 15.9 & 3.6 & 1.1 & 30.8 & 8.3 \\
& 10.5 & 4.0 & 0.8 & 28.8 & 5.8 \\
\hline & & & & &
\end{tabular}

Notes.

a Note that $\theta_{i}$ angles describe the orientation of the deduced magnetic field. The angles are measured relative to north and increasing eastward.

${ }^{b}$ Offset positions with respect to the peak position of NGC 1333 IRAS 4B.

${ }^{c}$ Data given below the solid line describe vectors associated with $4 \mathrm{~A}$.

discussion of the full implications of this finding on the role of rotational support in the formation of $4 \mathrm{~A}$ is beyond the scope of this paper. One would expect to see a significant deviation in the field morphology from small to large spatial scales if centrifugal forces were dominant in this system (Machida et al. 2006). Instead, the polarimetry results presented here plus those obtained at smaller spatial scales (G2006) indicate otherwise. Nevertheless, the presence of a binary system within 4A shows centrifugal forces could not be negligible in the formation of this system. All of this information is consistent with the idea that the magnetic and centrifugal forces were comparable in magnitude for this system during the onset of collapse (G2006). In addition, it should be noted that the large-scale uniform magnetic field implied by our results is aligned with the original (i.e., largescale) orientation of the outflow from $4 \mathrm{~A}$.

Finally, we wish to calculate the mean intrinsic dispersion angle $\left(\delta \theta_{\text {int }}\right)$ over $4 \mathrm{~A}$ by comparing our observations with the adopted uniform magnetic field model with $\theta \approx 45.9$. Now $\delta \theta_{\text {int }}$ is given by $\delta \theta_{\text {int }}^{2}=\delta \theta_{\text {obs }}^{2}-\sigma_{\theta}^{2}$, where $\sigma_{\theta}$ is the uncertainty in the observed position angle. We calculate values of $\delta \theta_{\text {obs }} \approx 13.6$ and $\sigma_{\theta} \approx 7.7$ and as such work out $\delta \theta_{\text {int }}$ to be $\approx 11$.2. We will employ this value in our general discussion in Section 3. Note that we do not attempt a similar analysis with our results over 4B. It is apparent from Figure 1 that we cannot fit our vectors over this source to a simple model for the field orientation (plus no polarization is detected over the peak flux of this source). As such, it is not possible to calculate a meaningful average field orientation $\theta$ or intrinsic dispersion $\delta \theta_{\text {int }}$ for $4 \mathrm{~B}$.

\subsection{Spectroscopy}

Observations of the $\mathrm{HCN} J=4 \rightarrow 3(354.505 \mathrm{GHz})$ rotational transition from $4 \mathrm{~A}$ were made with the CSO 300 $400 \mathrm{GHz}$ heterodyne receiver. The beam size at this frequency 


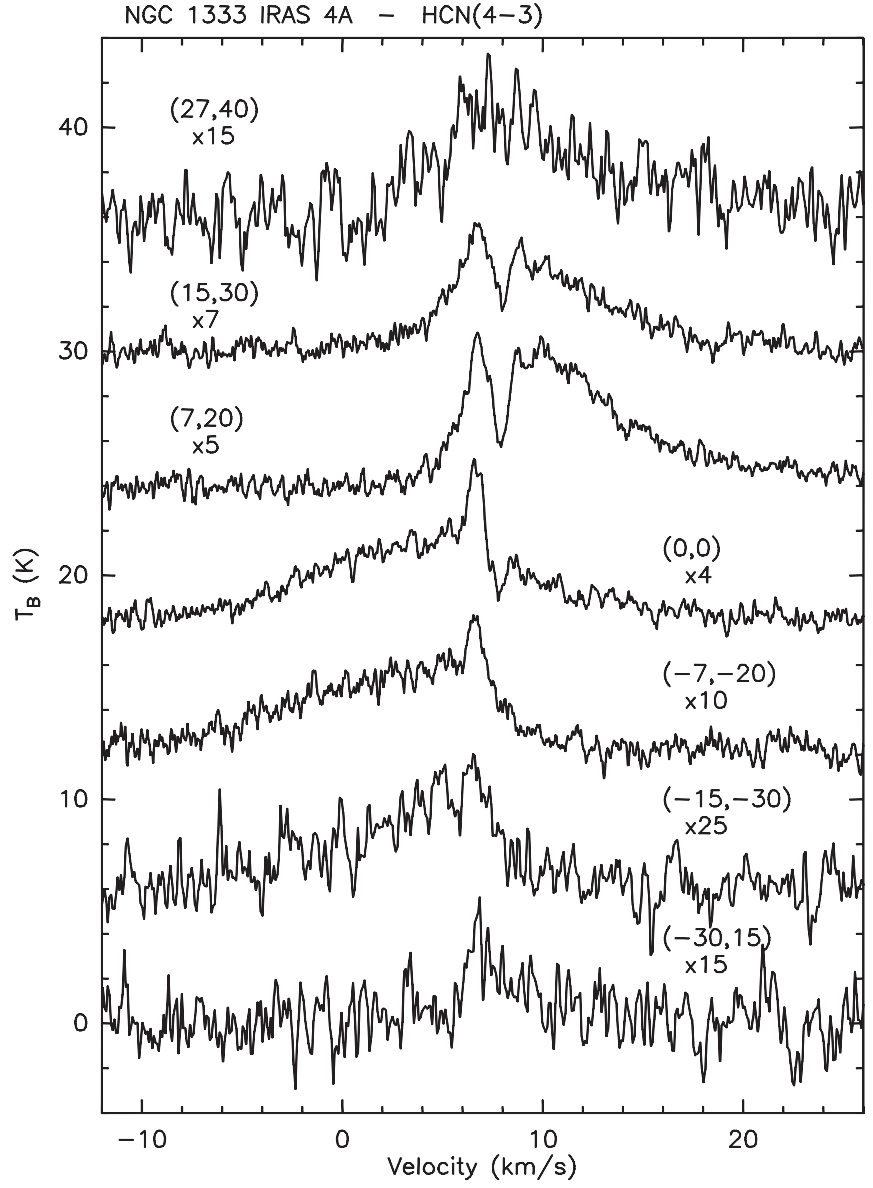

Figure 2. HCN $J=4 \rightarrow 3$ line emission $(354.505 \mathrm{GHz}$ ) centered on $4 \mathrm{~A}$

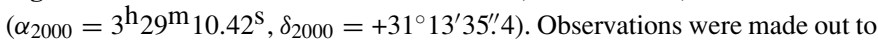
offset positions of $\left(\alpha_{o}=-15^{\prime \prime}, \delta_{o}=-30^{\prime \prime}\right)$ on the blue lobe of the outflow and $\left(\alpha_{o}=27^{\prime \prime}, \delta_{o}=40^{\prime \prime}\right)$ on the red lobe. One observation at an offset position of $\left(\alpha_{o}=-30^{\prime \prime}, \delta_{o}=15^{\prime \prime}\right)$ was made perpendicular to the outflow axis.

is approximately $20^{\prime \prime}(0.029)$ and thus samples a far larger region of space than was obtained with DF2001. Detections were obtained for a sequence of points lying approximately along the outflow axis of $4 \mathrm{~A}$, as well as for a single point that was displaced from the center in the perpendicular direction. In total, seven different positions were looked at; the results are illustrated in Figure 2.

What is immediately clear is the presence of a peak followed by a dip in the line emission centered on $4 \mathrm{~A}$ and the two positions lying closest to it along the red lobe of the outflow. We should note that the presence of the red-lobe outflow will distort our spectra, as the background that our source absorbs against is not flat but the outflow emission itself. Therefore, an interpretation of our data becomes clearer once the outflow component of the spectra is fitted with a Gaussian and removed, as was done using the CLASS software package and is shown in Figure 3. The spectra in Figure 3 are characterized solely by the aforementioned emission peak and dip, with the dip being situated on the right-hand side of the peak in each case. Each maxima and minima is observed at velocities of $6.8 \mathrm{~km} \mathrm{~s}^{-1}$ and $8.0 \mathrm{~km} \mathrm{~s}^{-1}$, respectively. The full width at half-maximum of each peak and dip profile is approximately $\simeq 1.5 \mathrm{~km} \mathrm{~s}^{-1}$, and the point between the maxima and minima where the line temperature $T_{B}$ equals $\approx 0 \mathrm{~K}$ corresponds to a velocity of approximately $V \approx 7 \mathrm{~km} \mathrm{~s}^{-1}$ in each case. These data have exactly the same characteristics as the $\mathrm{H}_{2} \mathrm{CO} 3_{12} \rightarrow 2_{11}$ spectra

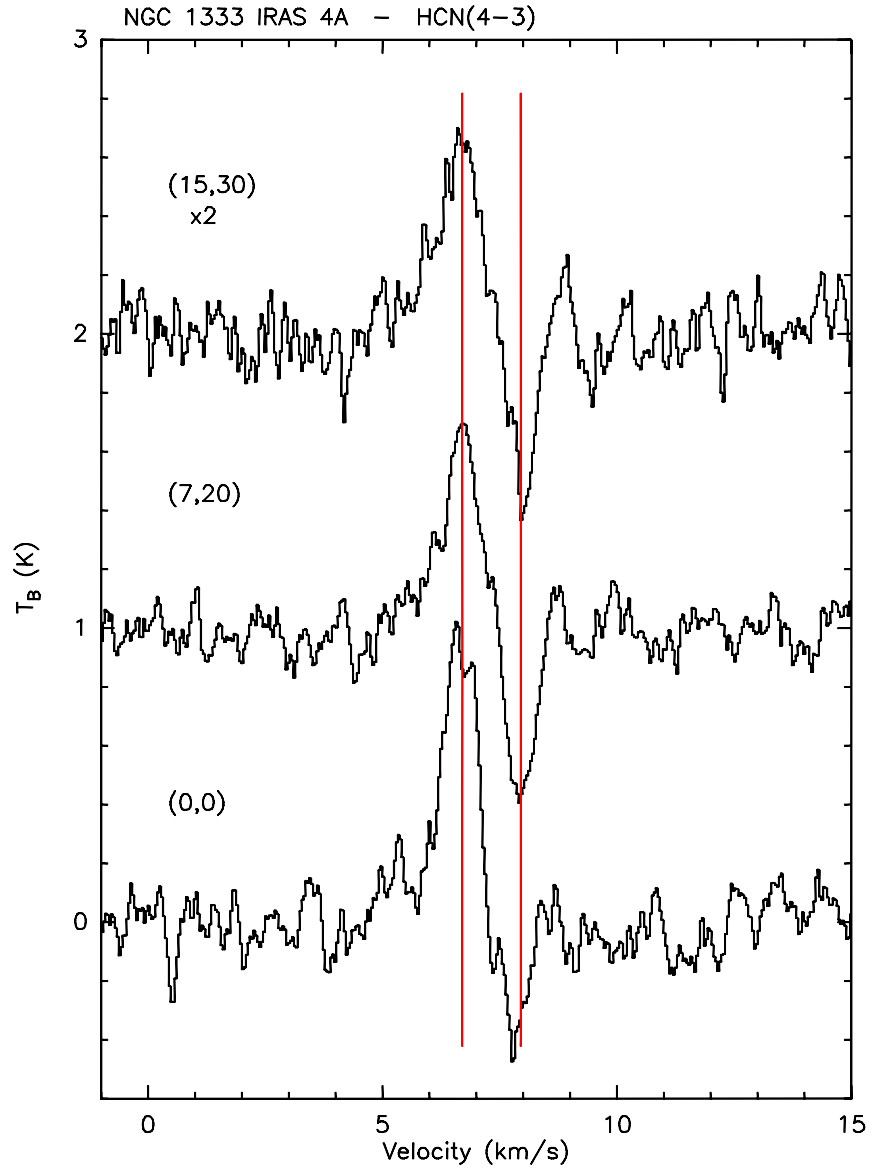

Figure 3. $\mathrm{HCN} J=4 \rightarrow 3$ line emission at offset positions $\left(\alpha_{o}=0^{\prime \prime}, \delta_{o}=0^{\prime \prime}\right)$, $\left(\alpha_{o}=7^{\prime \prime}, \delta_{o}=20^{\prime \prime}\right)$, and $\left(\alpha_{o}=15^{\prime \prime}, \delta_{o}=30^{\prime \prime}\right)$ from the peak flux of 4A. Outflow components have been removed to reveal characteristic inverse P Cygni profiles at each of the three locations. The outflows were removed with the use of the CLASS software package. The vertical lines represent the velocities at $6.8 \mathrm{~km} \mathrm{~s}^{-1}$ and $8.0 \mathrm{~km} \mathrm{~s}^{-1}$.

(A color version of this figure is available in the online journal.)

presented in Figure 4 of DF2001 and are thus the characteristic of inverse P Cygni profiles. With this interpretation, we note that the spectral signatures seen in Figure 2 are the characteristic of an infalling envelope of gas around 4A plus a outflow. These features are not observed toward the blue lobe of the outflow. This is to be expected due to the position of the blue-lobe outflow, which is situated in between the infalling envelope and the observer. Therefore, this component of the outflow does not provide a background against which the infalling material can absorb. We do not have spectroscopy data on 4B at this time, and as such we cannot comment on the nature of gas motions around this core.

One also notices the disappearance of the inverse P Cygni profile at an offset position of $\alpha_{o}=27^{\prime \prime}, \delta_{o}=40^{\prime \prime}$ along the red-lobe outflow. We can therefore state that the peak of $4 \mathrm{~A}$ is surrounded by an infalling envelope of radius $r_{c}$ on the plane of the sky, where $0.05 \leqslant r_{c}<0.07$. The lower bound of $r_{c}$ is provided by the spectra obtained at an offset position of $\alpha_{o}=15^{\prime \prime}$ and $\delta_{o}=30^{\prime \prime}$ - the furthest position away from the peak of $4 \mathrm{~A}$ at which the infall signature is still clearly apparent. The range of $r_{c}$ specified here is consistent with the value of $\approx 0.1$ predicted by models of self-gravitating cores (McKee \& Ostriker 2007). Note that this estimate relies upon the assumption that nothing obstructs our view of the infall signature 

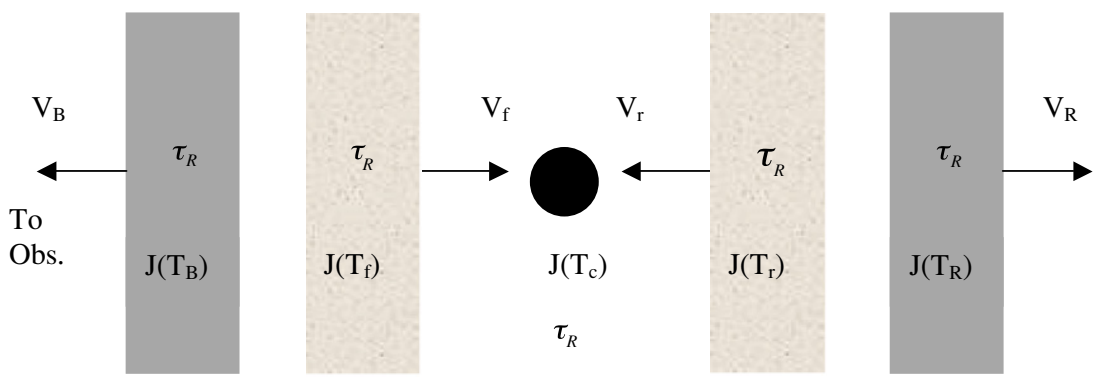

Figure 4. Illustration of the enhanced "four-layered" version of the two-layered model of Myers et al. (1996). This depiction labels each component of the model with a Planck temperature $J\left(T_{i}\right)$, optical depth $\tau_{i}$, and a velocity $V_{i}$. The subscript $i$ denotes " $B$ ", " $R$ ", " $f$ ", " $r$ ", and " $c$ " representing the blue outflow, red outflow, front slab, rear slab, and central source parameters, respectively. The arrows indicate velocity directions along the observers line of sight. We also allow for cosmic background radiation with a Planck temperature $J\left(T_{b g}\right)=0.49 \mathrm{~K}$.

at a radial distance $\approx 0.07$ from the peak flux of $4 \mathrm{~A}$. Adopting $r_{c} \approx 0.06$, we immediately note that the size of the infalling envelope is approximately $\sim 4$ times larger than the upper limit placed on the size of the magnetic pinch observed around 4A (see Section 2.1). This result is consistent with theoretical work on the collapse of magnetized cloud cores (Galli \& Shu 1993).

\section{DISCUSSION}

\subsection{Kinematics}

To further our investigation of the 4A system, we fit our three inverse P Cygni profile detections to an enhanced version of the "two-layer" model originally devised by Myers et al. (1996) and later used by DF2001. In its most general form, our treatment of this problem is to envisage two parallel layers of material moving toward an opaque central condensation. These two layers, denoted "front slab" and "rear slab," represent the infalling envelope. The opaque source is taken to fill a fraction $\Upsilon$ of the telescope beam. This setup is the model of DF2001, where the presence of outflows was not accounted for. For our model, two more layers of material are included in this system to represent the blue- and red-lobe outflows. This scenario is illustrated in Figure 4.

Quantifying this model into an expression of the line brightness temperature $T_{\mathrm{B}}(V)$ at a certain velocity $V$ we obtain Equation (1) below. Note the subscripts " $B$ ", " $R$ ", " $f$ ", " $r$ ", " $c$ ", and " $b g$ " represent the blue-lobe outflow, red-lobe outflow, front slab, rear slab, central source, and cosmic background parameters, respectively. Therefore, the analytical model for the scenario depicted in Figure 4 is

$$
\begin{aligned}
T_{\mathrm{B}}(V)= & (1-\Upsilon) \cdot J\left(T_{R}\right) \cdot\left(1-e^{-\tau_{R}}\right) \cdot e^{-\tau_{0}}+(1-\Upsilon) \\
& \cdot J\left(T_{r}\right) \cdot\left(1-e^{-\tau_{r}}\right) \cdot e^{-\tau_{f}-\tau_{B}} \\
& +J\left(T_{f}\right) \cdot\left(1-e^{-\tau_{f}}\right) \cdot e^{-\tau_{B}}+J\left(T_{B}\right) \cdot\left(1-e^{-\tau_{B}}\right) \\
& -\Upsilon \cdot J\left(T_{c}\right) \cdot\left(1-e^{-\tau_{f}-\tau_{B}}\right) \\
& -(1-\Upsilon) \cdot J\left(T_{b g}\right) \cdot\left(1-e^{-\tau_{\star}}\right),
\end{aligned}
$$

where $J(T)=T_{0} /\left(e^{T_{0} / T}-1\right)$ is the Planck temperature as a function of the blackbody temperature $T$, and $T_{0}=h v / k$, where $h$ is the Planck constant, $k$ is the Boltzmann constant, and $v$ is the frequency. The optical depth for the model component $i$ is denoted as $\tau_{i}$. Finally, we note that $\tau_{0}=\tau_{r}+\tau_{f}+\tau_{B}$ and $\tau_{\star}=\tau_{R}+\tau_{0}$. Following Myers et al. (1996), we model the different optical depths with Gaussian profiles:

$$
\tau_{i}=\tau_{0 i} \cdot \exp \left[\frac{-\left(V-V_{i}-V_{\mathrm{LSR}}\right)^{2}}{2 \sigma_{i}^{2}}\right]
$$

$$
\tau_{j}=\tau_{0 j} \cdot \exp \left[\frac{-\left(V+V_{j}-V_{\mathrm{LSR}}\right)^{2}}{2 \sigma_{j}^{2}}\right],
$$

where $i=f, R$ and $j=r, B, \tau_{0 i}$ and $\tau_{0 j}$ are the peak optical depths, $\sigma_{i}$ and $\sigma_{j}$ are the respective velocity dispersions, and the velocity for the local standard of rest is taken to be $V_{\text {LSR }} \approx 6.96 \mathrm{~km} \mathrm{~s}^{-1}$ (DF2001). Note that $V_{f}$ and $V_{r}$ are the infall velocities for the front and rear slabs, respectively.

We can simplify (1) by making note of two important properties of our data. First, our large beam size implies $\Upsilon \approx 0$ (DF2001 get a value of $\Upsilon \approx 0.3$ with a $2^{\prime \prime}$ beam). Second, we neglect the contribution of the blue lobe, as only the red-lobe component of the outflow provides the background radiation against which the infalling material can absorb. It is clear from Figure 2 that the spectra centered on $\left(\alpha_{o}=0^{\prime \prime}, \delta_{o}=0^{\prime \prime}\right)$ has a significant blue-lobe outflow component, while the spectra centered on $\left(\alpha_{o}=7^{\prime \prime}, \delta_{o}=20^{\prime \prime}\right)$ and $\left(\alpha_{o}=15^{\prime \prime}, \delta_{o}=30^{\prime \prime}\right)$ are largely dominated by the red-lobe outflow. As such, our outflow approximation will be especially coarse in the case of the spectra centered on the peak flux of $4 \mathrm{~A}$. By setting $\Upsilon=0$ and $\tau_{B}=0$ in Equation (1), we get

$$
\begin{aligned}
T_{\mathrm{B}}\left(V, \tau_{B}=0\right)= & J\left(T_{R}\right) \cdot\left(1-e^{-\tau_{R}}\right) \cdot e^{-\tau_{f}-\tau_{r}} \\
& +J\left(T_{r}\right) \cdot\left(1-e^{-\tau_{r}}\right) \cdot e^{-\tau_{f}}+J\left(T_{f}\right) \\
& \cdot\left(1-e^{-\tau_{f}}\right)-J\left(T_{b g}\right) \cdot\left(1-e^{-\tau_{\oplus}}\right),
\end{aligned}
$$

where $\tau_{\oplus}=\tau_{f}+\tau_{r}+\tau_{R}$. A computer program was written to minimize the reduced $-\chi^{2}$ function generated by comparing Equation (4) with a multi-Gaussian fit to the three aforementioned spectra in Figure 2 that exhibit inverse P Cygni profiles. The minimization was carried out through the use of Powell's method for multidimensional functions (Press et al. 2002, p. 412). The resulting fit of Equation (4) to our spectra at offset positions $\left(\alpha_{o}=0^{\prime \prime}, \delta_{o}=0^{\prime \prime}\right),\left(\alpha_{o}=7^{\prime \prime}, \delta_{o}=20^{\prime \prime}\right)$, and $\left(\alpha_{o}=15^{\prime \prime}, \delta_{o}=30^{\prime \prime}\right)$ is shown in Figure 5.

The plots shown in Figure 5 demonstrate a reasonably good agreement between the actual data and the model. We should take stock at this point to stress that this model provides the simplest possible explanation for a contracting system with outflow. As such, it will provide us with only an approximate picture of the physical properties at play in $4 \mathrm{~A}$ and its surroundings. This is especially true with regard to the optical depths and temperatures of the absorbing/emitting gas (DF2001). It should also be mentioned that the resolution of our data prevents us from distinguishing between small-scale structures, such as the binary system at the core of the 4A complex (dubbed 4A1 and 4A2, G2006). Although we dispense with the optically thick central 

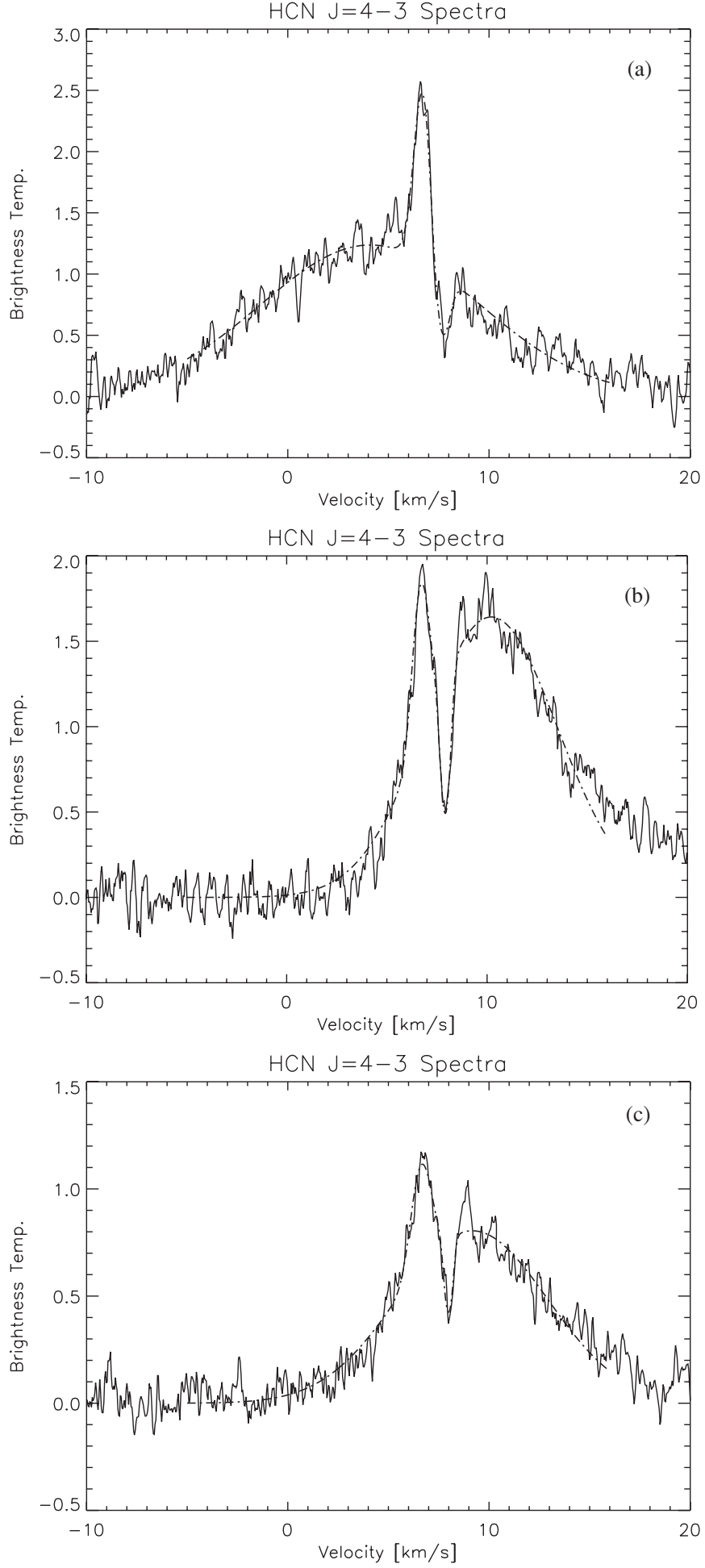

Figure 5. HCN $J=4 \rightarrow 3$ line emission observed at offset positions (a) $\left(\alpha_{o}=0^{\prime \prime}, \delta_{o}=0^{\prime \prime}\right)$, (b) $\left(\alpha_{o}=7^{\prime \prime}, \delta_{o}=20^{\prime \prime}\right)$, and (c) $\left(\alpha_{o}=15^{\prime \prime}, \delta_{o}=30^{\prime \prime}\right)$ from the peak continuum flux of 4A. The dashed curves show the fit of our model (Equation (4) in the text).

condensation in Equation (4), each member of the binary system drives a unique outflow (Choi 2005). ${ }^{9}$ By far the dominant outflow originates from $4 \mathrm{~A} 2$, which possesses a length $\sim 11$ times longer and is more luminous than the outflow associated with

\footnotetext{
9 Note that all referrals to the "4A outflow" made elsewhere in this paper pertain to the one driven by $4 \mathrm{~A} 2$.
}

4A1 (Choi 2005; Blake et al. 1995). Our spectra likely sample an average of the environment of $4 \mathrm{~A}$, with detections of both outflows being integrated for data obtained on the peak flux of 4A. However, it is unlikely that the data obtained away from the peak will be affected by the outflow of $4 \mathrm{~A} 1$, since this outflow is limited in extent and has a position angle shifted by $\sim 20^{\circ}$ to the position angle of the much larger 4A2 outflow. Despite these shortcomings, our model is still useful for the comparison of the infall velocity between different spectra, since the ratio $V_{f} / \sigma_{f}$ (or $V_{r} / \sigma_{r}$ ) is a key parameter for any model of a contracting system (DF2001; Leung \& Brown 1977).

The model parameters resulting from the simulations shown in Figure 5 are listed in Table 2. One notices immediately that $V_{f}>V_{r}$ for each scan, where the values of $V_{f}$ tend to be close to a speed of $\sim 1 \mathrm{~km} \mathrm{~s}^{-1}$, while $V_{r}$ tends to have values closer to $\sim 0.3 \mathrm{~km} \mathrm{~s}^{-1}$. A likely explanation for this is that the value of $V_{\text {LSR }}$ set in our model is somewhat inaccurate, and thus introduces a systematic error in our calculations. We note that DF2001 leave $V_{\mathrm{LSR}}$ as a free parameter in their version of the "two-layered" model. Our attempt to treat $V_{\mathrm{LSR}}$ as a free parameter in Equation (4) failed to produce satisfactory results. This may be due to the highly nonlinear nature of our model, which makes an iterative fit of Equation (4) to a particular data set very sensitive to the initial parameter values. The simplest means to proceed was by fixing $V_{\mathrm{LSR}}$ to the DF2001 value. The differences between Equation (4) and the DF2001 model may account for the different $V_{\mathrm{LSR}}$ value in our case. To correct for this, we take an arithmetic average of the $V_{f}$ and $V_{r}$ values listed in Table 2, which result in mean infall velocities of $0.63 \mathrm{~km} \mathrm{~s}^{-1}, 0.61 \mathrm{~km} \mathrm{~s}^{-1}$, and $0.67 \mathrm{~km} \mathrm{~s}^{-1}$ for the scans at offset positions $\left(\alpha_{o}=0^{\prime \prime}, \delta_{o}=0^{\prime \prime}\right),\left(\alpha_{o}=7^{\prime \prime}\right.$, $\left.\delta_{o}=20^{\prime \prime}\right)$, and $\left(\alpha_{o}=15^{\prime \prime}, \delta_{o}=30^{\prime \prime}\right)$, respectively. Taking all three scans together we estimate a mean infall velocity of $\approx 0.64 \mathrm{~km} \mathrm{~s}^{-1}$ for $4 \mathrm{~A}$, a value that is very similar to the $0.68 \mathrm{~km} \mathrm{~s}^{-1}$ velocity calculated by DF2001. If we assume a gas temperature of $T_{g} \approx 30 \mathrm{~K}$ (Blake et al. 1995), we can calculate the isothermal sound speed $V_{\mathrm{rms}}=\sqrt{k T_{g} / \mu m_{\mathrm{H}}}$ to be $\approx 0.33 \mathrm{~km} \mathrm{~s}^{-1}$, where $k$ is Boltzmann's constant, $\mu$ is the mean molecular weight $(\mu=2.22)$, and $m_{\mathrm{H}}$ is the mass of hydrogen. Thus, the observed infall is also supersonic.

Assuming inside-out collapse for $4 \mathrm{~A}$, we note that the radius $r_{c}$ of the expansion wave for the infalling envelope is given by $r_{c}=V_{\mathrm{rms}} \times t$, where $V_{\mathrm{rms}}$ is defined above and $t$ is the time since the onset of collapse. Taking $r_{c} \approx 0.06$ (see Section 2.2), we find $t \approx 2 \times 10^{5}$ yr. A large degree of uncertainty exists with regard to the age of the outflow, but estimates range from 2000 to $20000 \mathrm{yr}$ (Blake et al. 1995). Therefore, the age of the infalling envelop in $4 \mathrm{~A}$ is roughly $10-100$ times the age of the associated outflow.

Outflow velocities along the line of sight of $-2.86 \mathrm{~km} \mathrm{~s}^{-1}$, $10.21 \mathrm{~km} \mathrm{~s}^{-1}$, and $9.17 \mathrm{~km} \mathrm{~s}^{-1}$ are estimated from the fits of Equation (4) to the scans at offset positions $\left(\alpha_{o}=0^{\prime \prime}\right.$, $\left.\delta_{o}=0^{\prime \prime}\right),\left(\alpha_{o}=7^{\prime \prime}, \delta_{o}=20^{\prime \prime}\right)$, and $\left(\alpha_{o}=15^{\prime \prime}, \delta_{o}=30^{\prime \prime}\right)$, respectively. The latter two values indicate the red-lobe outflow has an approximate velocity of $\approx 10 \mathrm{~km} \mathrm{~s}^{-1}$. The negative value for the velocity at position $\left(\alpha_{o}=0^{\prime \prime}\right.$ and $\left.\delta_{o}=0^{\prime \prime}\right)$ is due to the fact that Equation (4) treats for a red-lobe outflow only, while the spectrum at this position shows a significant blue-lobe outflow as well. The velocity values for both red-lobe outflows are approximately consistent with $\mathrm{SiO} J=1 \rightarrow 0$ observations presented in Choi (2005) and illustrated in Figure 5 of that paper. The outflow observed at position $\left(\alpha_{o}=0^{\prime \prime}\right.$ and $\left.\delta_{o}=0^{\prime \prime}\right)$ is a 
Table 2

Model Parameters for $\mathrm{HCN} J=4 \rightarrow 3$ Inverse P Cygni Profiles $^{\mathrm{b}}$

\begin{tabular}{lcccccccccccc}
\hline \hline$\alpha_{o}, \delta_{o}{ }^{\mathrm{a}}$ & $J\left(T_{f}\right)(\mathrm{K})$ & $J\left(T_{r}\right)(\mathrm{K})$ & $\tau_{0 f}$ & $\tau_{0 r}$ & $V_{f}\left(\mathrm{~km} \mathrm{~s}^{-1}\right)$ & $\sigma_{f}\left(\mathrm{~km} \mathrm{~s}^{-1}\right)$ & $J\left(T_{R}\right)(\mathrm{K})$ & $V_{R}\left(\mathrm{~km} \mathrm{~s}^{-1}\right)$ & $\sigma_{R}\left(\mathrm{~km} \mathrm{~s}^{-1}\right)$ & $\tau_{R}$ & $V_{r}\left(\mathrm{~km} \mathrm{~s}^{-1}\right)$ & $\sigma_{r}\left(\mathrm{~km} \mathrm{~s}^{-1}\right)$ \\
\hline $0^{\prime \prime}, 0^{\prime \prime}$ & 0.30 & 11.00 & 0.49 & 0.15 & 0.95 & 0.36 & 107.14 & -2.86 & 5.44 & 0.01 & 0.30 & 0.44 \\
$7^{\prime \prime}, 20^{\prime \prime}$ & 0.37 & 4.34 & 0.84 & 0.37 & 0.96 & 0.22 & 128.59 & 10.21 & 3.29 & 0.01 & 0.26 & 0.40 \\
$15^{\prime \prime}, 30^{\prime \prime}$ & 0.75 & 2.50 & 1.15 & 0.42 & 1.03 & 0.18 & 118.57 & 9.17 & 3.72 & 0.01 & 0.31 & 0.47 \\
\hline
\end{tabular}

Notes.

${ }^{\text {a }}$ Offset positions with respect to the peak position of NGC 1333 IRAS 4A.

${ }^{\mathrm{b}}$ Variable definitions are given in Section 3.1.

composite of both the red- and blue-lobes and hence our model does not provide a reliable outflow velocity for this case.

As a final note, we wish to briefly discuss our handling of the outflow velocity $V_{R}$ employed in our model for the scan at position $\left(\alpha_{o}=0^{\prime \prime}\right.$ and $\left.\delta_{o}=0^{\prime \prime}\right)$. Because of the simplifications introduced into Equation (4), where only the red-lobe outflow is included analytically, it is necessary to set this parameter to a negative value in order to fit the composite red- and bluelobe outflows that are present in this spectrum. Although this points out a shortcoming of our model, the important point here is to maintain emitting gas in the background of the infalling envelope - a red-lobe outflow. This component of the model provides emission from a backdrop of material against which the infalling gas can absorb and thus produce the dip present in an inverse $\mathrm{P}$ Cygni profile. This is the reason why $V_{R}$ is negative in Equation (4) at position $\left(\alpha_{o}=0^{\prime \prime}\right.$ and $\left.\delta_{o}=0^{\prime \prime}\right)$. Despite this obvious defect, the model used here is the simplest mathematical construct that still conveys physical meaning for the data at hand. Future work may wish to employ more sophisticated Monte Carlo techniques to generate models that more accurately describe this source.

\subsection{Support Mechanism}

We now proceed to calculate a mass estimate for $4 \mathrm{~A}$ and $4 \mathrm{~B}$. This can be achieved through a careful treatment of the thermal dust continuum data from SHARP. The mass of a cloud can be estimated via its submillimeter thermal emission by

$$
M=\frac{4}{3} \varrho d^{2} \frac{F_{v}}{B_{v}\left(T_{d}\right)} \frac{a}{\varepsilon_{v}} R,
$$

where $R$ is the gas-to-dust ratio, $\varrho$ is the density of the dust material, $d$ is the distance to the cloud, $F_{v}$ is the measured flux density, $B_{v}\left(T_{d}\right)$ is the Planck function with dust temperature $T_{d}$, $a$ is the grain radius, and $\varepsilon_{v}$ is the dust emissivity (Hildebrand 1983). Here we assume the values of $R=100, \varrho=3 \mathrm{~g} \mathrm{~cm}^{-3}$, $d=300, a=10^{-5} \mathrm{~cm}, \varepsilon_{v}=2.26 \times 10^{-4}$, and $T_{d}=50 \mathrm{~K}$, where the dust temperature was chosen to be the same value as that employed in G2006. The values of $\varrho, a$, and $\varepsilon_{v}$ were taken or inferred from Hildebrand (1983). The total amount of $350 \mu \mathrm{m}$ flux detected within a radial distance of $20^{\prime \prime}(0.03)$ from $4 \mathrm{~A}$ is $\approx 179 \mathrm{Jy}$, while a total of $\approx 76 \mathrm{Jy}$ was detected within $10^{\prime \prime}(0.015)$ of $4 \mathrm{~B}$. The distance around 4A was selected to encompass the polarimetry vectors used in the calculation of $\delta \theta_{\text {int }}$ in Section 2.1. With this information, the mass of $4 \mathrm{~A}$ and $4 \mathrm{~B}$ is estimated to be $\approx 1.9 M_{\odot}$ and $\approx 0.8 M_{\odot}$, respectively. These values are comparable to the mass estimates of DF2001 and G2006 that are stated in Section 1 of this work. As a final point, we note that the total flux detected in Figure 1 is $\approx 511 \mathrm{Jy}$. From this flux value a total detected mass of $\approx 5.4 M_{\odot}$ is calculated for the area of NGC 1333 IRAS 4 mapped by our continuum observations.
We can now apply a modified form of the Chandrasekhar \& Fermi (CF) technique (Chandrasekhar \& Fermi 1953) to obtain an estimate of the magnetic field strength around 4A. If we assume the dispersion in our magnetic field map (see Figure 1(b)) is entirely due to Alfvén waves and/or turbulence, then the plane-of-sky magnetic field strength will be given by

$$
B_{\mathrm{pos}}=Q\left(\frac{\delta v_{\mathrm{los}}}{\delta \theta_{\text {int }}}\right)(4 \pi \rho)^{1 / 2} .
$$

Here we take $Q=0.5$, the same value used in G2006 and one that is indicated by simulations of turbulent molecular clouds (Ostriker et al. 2001). We have already calculated $\delta \theta_{\text {int }} \approx 11.2$ in Section 2 . The gas density $\rho$ can be roughly estimated by dividing the mass of $4 \mathrm{~A}$ calculated above by the volume of a $20^{\prime \prime}(0.03)$ radius sphere. Doing this yields a density of $\rho \approx 8 \times 10^{-19} \mathrm{~g} \mathrm{~cm}^{-3}$. The line-of-sight velocity dispersion $\delta v_{\text {los }}$ is taken from observations of $\mathrm{HCO}^{+} J=4 \rightarrow 3$ obtained at the same time (and using the same heterodyne receiver) as the HCN observations discussed earlier. These data were obtained while pointing on the peak flux of $4 \mathrm{~A}$ and are shown in Figure 6. The choice of analyzing this spectrum for the $\delta v_{\text {los }}$ value is motivated by the fact that an ion will be better coupled to the magnetic field (and the dust) than a neutral species over the whole turbulent energy density spectrum (Li \& Houde 2008). Thus, the line width of this species will be more representative of the turbulence/Alfvén waves that may be disturbing the field as opposed to a neutral molecular counterpart. The value of $\delta v_{\text {los }}$ is found to be $1.67 \mathrm{~km} \mathrm{~s}^{-1}$. Inserting this information into Equation (6) yields a field strength of $B_{\text {pos }} \approx 1.4 \mathrm{mG}$, a value that is roughly a factor of 3.6 lower than the G2006 result. This difference is not unexpected since the field strength should increase toward the center of the core, as this is the location of maximum compression of the field lines. One could thus expect the value of $B_{\text {pos }}$ to diminish at larger spatial scales.

We are now in a position to calculate the mass-to-flux ratio for our observations on $4 \mathrm{~A}$. Writing this quantity in terms of the critical value for collapse $\lambda$, we find

$$
\lambda=\frac{M / \Phi}{c_{\Phi} / \sqrt{G}},
$$

where $c_{\Phi}=0.12$ (Tomisaka et al. 1988), $\Phi$ is the magnetic flux, and $G$ is the gravitational constant. Using this relation, we calculate $\lambda \approx 0.44$ over a region of $\approx 270 \operatorname{arcsec}^{2}$ centered on the peak of $4 \mathrm{~A}$. This value is a factor of $\approx 2.3$ lower than unity and also notably lower than the G2006 result. A strongly subcritical cloud is also inconsistent with our own observations of infalling gas motions (Section 2.2). Uncertainties in our estimate of $M$ may be partly responsible; submillimeter continuum emission will fail to sample the hot protostellar mass where dust particles cannot exist. We should note that the use of 


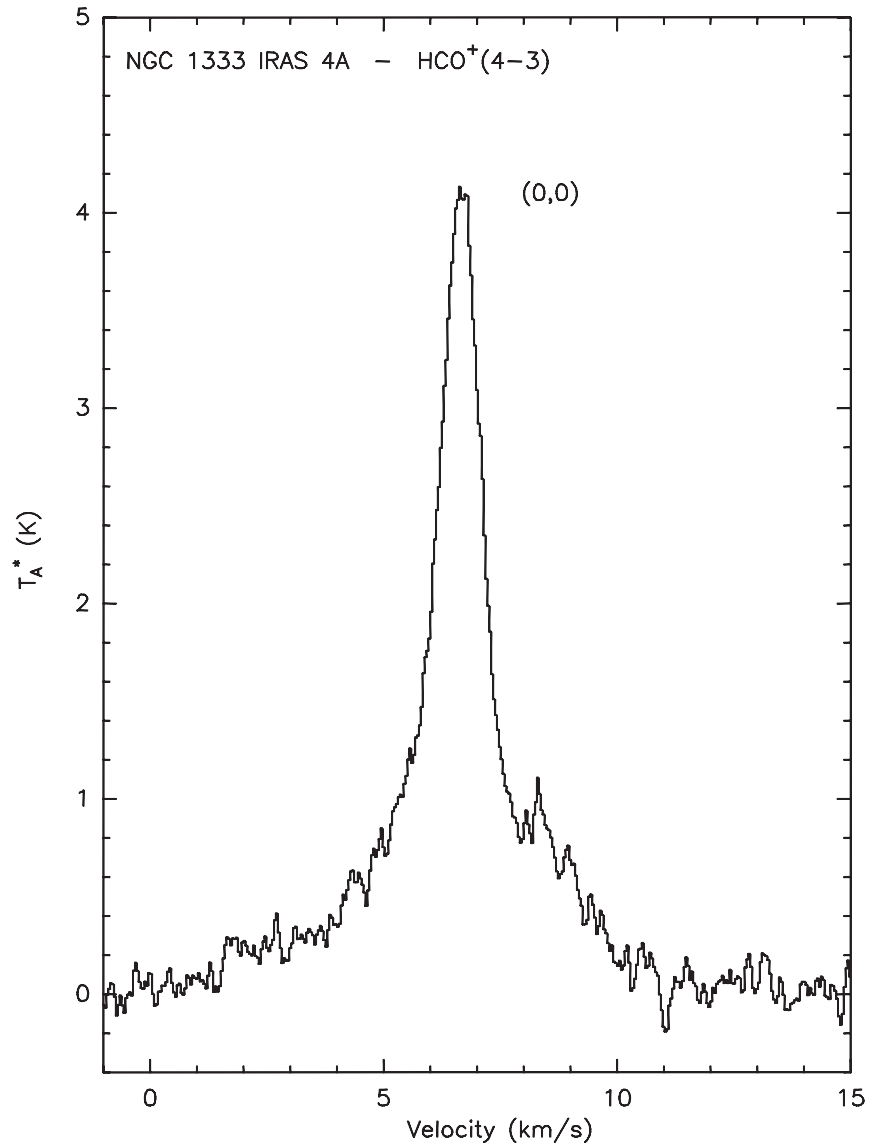

Figure 6. $\mathrm{HCO}^{+} J=4 \rightarrow 3$ line emission at an offset position of ( $\alpha_{o}=0^{\prime \prime}$, $\left.\delta_{o}=0^{\prime \prime}\right)$ from the peak continuum flux of 4A. These data were used for the evaluation of $\delta v_{\text {los }}$ in our calculation of $B_{\text {pos }}$. We have assumed that the ion will be more closely associated with the magnetic field through the Lorentz force, and hence the line width observed here should be more representative of the turbulence/MHD waves that may disturb the embedded field. The line width is calculated to be $\delta v_{\text {los }} \approx 1.67 \mathrm{~km} \mathrm{~s}^{-1}$

the plane-of-sky component of the magnetic field will result in an underestimate of $\Phi$ and thus lead to a overestimation of $\lambda$. Recent work suggests that the inclination angle $\vartheta$ of the embedded magnetic field in this cloud falls within the range $0^{\circ} \leqslant \vartheta \leqslant 55^{\circ}$ (Goncalves et al. 2008). This suggests that the magnitude of $B=B_{\text {pos }} / \cos \vartheta$ could fall somewhere within $1.4 \mathrm{mG} \leqslant B \leqslant 2.4 \mathrm{mG}$. Therefore, we can speculate that this effect could result in an overestimation of $\lambda$ by up to a factor of $\approx 1.7$.

A more likely explanation for the sub-critical value of $\lambda$ may come from the application of the $\mathrm{CF}$ technique itself. Crucial to this calculation is an accurate measure of the intrinsic dispersion angle $\delta \theta_{\text {int }}$ of the magnetic field vectors. Polarimetry, however, does not distinguish between contributions along the line of sight; the result being that the angular dispersion will be reduced through the process of signal integration through the thickness of the cloud as well as across the area subtended by the telescope beam (Hildebrand et al. 2009). The value of $\delta \theta_{\text {int }}$ that is then calculated could be smaller than the true dispersion and thus may result in an overestimation of $B_{\text {pos }}$ through Equation (6). Despite the fact that the factor $Q$ in Equation (6) is meant to account for the problem of signal integration through the cloud (Ostriker et al. 2001), in reality this is only a first-order correction and the smoothing effect could be more severe (Houde et al. 2009). Higher resolution polarimetry across the large spatial scales observed here in conjunction with a more in-depth analysis may be used in the future to resolve this issue (Hildebrand et al. 2009; Houde et al. 2009).

We can now calculate the ratio of the turbulent to magnetic energy $\left(\beta_{\text {turb }}\right)$ in $4 \mathrm{~A}$. The value we determine for this quantity is $\beta_{\text {turb }} \approx 8 \times 10^{-4}\left(\delta \theta_{\text {int }} / 1^{\circ}\right)^{2}=0.05$, suggesting that magnetic forces dominate the physics of this particular cloud. From our discussion above we note that the field strength calculated here could be overestimated by a factor of a few. This could result from an underestimate of the intrinsic dispersion in the polarimetry $\delta \theta_{\text {int }}$. For example, a requirement for a critical value of $\lambda$ would be an intrinsic dispersion value of $\delta \theta_{\text {int }} \approx 26^{\circ}$. This would then correspond to a value of $\beta_{\text {turb }} \approx 0.5$, a value sufficiently close to unity to suggest an equipartition of the turbulent and magnetic energies in this system. These results are definitely not consistent with the idea of a turbulentdominated support system for this cloud. Since models of magnetically supported star formation predict $\lambda \approx 1$ for cloud cores undergoing collapse, we conclude that our results are likely consistent with the idea that the turbulent and magnetic energies are of the same order of magnitude in this system.

\section{CONCLUSIONS}

We summarize our results as follows.

1. Dust continuum polarimetry done at $350 \mu \mathrm{m}$ with SHARP demonstrates a uniform magnetic field morphology around $4 \mathrm{~A}$ at a resolution scale of $10^{\prime \prime}(0.015)$. We therefore adopt the size of one resolution element to be an approximate upper limit on the size of the magnetic pinch region reported in G2006. This is in agreement with magnetic cloud collapse theory and correlates well with the findings of G2006. In addition, the large-scale uniform magnetic field appears to be aligned with the original (large-scale) outflow direction for 4A. Why the large-scale and smallscale directions of the 4A outflow are different is not known, but our results and those of G2006 show that the average orientation of the magnetic field has not changed direction from large-scales to small. The polarimetry obtained on 4B appears to indicate depolarization toward the peak flux region. An explanation for these observations on $4 \mathrm{~B}$ remains an open point. Mass estimates for both $4 \mathrm{~A}$ and 4B have been made revealing $1.9 M_{\odot}$ within $20^{\prime \prime}(0.03)$ and $0.8 M_{\odot}$ within $10^{\prime \prime}(0.015)$ of the peak flux for both cores, respectively. The total mass traced by our continuum observations is $5.4 M_{\odot}$ for the portion of the NGC 1333 IRAS 4 cloud complex surveyed.

2. Spectroscopy done at the CSO with HCN $J=4 \rightarrow 3$ line emission has revealed inverse P Cygni profiles at offset positions $\left(\alpha_{o}=0^{\prime \prime}, \delta_{o}=0^{\prime \prime}\right),\left(\alpha_{o}=7^{\prime \prime}, \delta_{o}=20^{\prime \prime}\right)$, and $\left(\alpha_{o}=15^{\prime \prime}, \delta_{o}=30^{\prime \prime}\right)$ from the location of peak flux for $4 \mathrm{~A}$. Fitting these data with an enhanced version of the Myers et al. (1996) "two-layer" model, we estimate a mean infall speed of $0.64 \mathrm{~km} \mathrm{~s}^{-1}$ for this cloud core. These findings are in good agreement with the results of DF2001. The radial size of the infalling envelope $r_{c}$ is estimated to range between $0.05 \leqslant r_{c}<0.07$ and is thus approximately $\sim 4$ times larger than the size of the magnetic pinch. This is consistent with theoretical work on magnetized cloud collapse (Galli \& Shu 1993). The age of the infalling envelope is found to be approximately $\approx 2 \times 10^{5} \mathrm{yr}-\mathrm{a}$ figure that is roughly 10-100 times larger than the age of the associated outflow. 
3. The value of the plane-of-sky component of the magnetic field strength has been estimated to be $\approx 1.4 \mathrm{mG}$ around $4 \mathrm{~A}$. This yields a normalized mass-to-flux ratio of $\lambda \approx 0.44$. This value of $\lambda$ is inconsistent with our observations of infalling gas motions and does not agree with the previous results of G2006. We speculate here that $\delta \theta_{\text {int }}$ may be underestimated due to smoothing effects in the angular dispersion along the line of sight that is the characteristic of polarimetry. If we inflate our value of $\delta \theta_{\text {int }}$ by a factor of $1 / \lambda$, we then find that $\beta_{\text {turb }} \approx 0.5$. This suggests an equipartition of the magnetic and turbulent energies in this cloud.

Our group is grateful for the assistance of the Caltech Submillimeter Observatory staff in installing and observing with SHARP and the heterodyne receiver. We also acknowledge the help provided by Jose Girart, Megan Krejny, Roger Hildebrand, Tristan Matthews, Larry Kirby, and Lerothodi Leeuw. M.A.'s and M.H.'s research is funded through the NSERC Discovery Grant, Canada Research Chair, Canada Foundation for Innovation, Ontario Innovation Trust, and Western's Academic Development Fund programs. SHARC II is funded through the NSF grant AST 05-40882 to the California Institute of Technology. The development of SHARP was funded by an NSF grant to Northwestern University (AST 02-43156), and its subsequent commissioning was funded by NSF grants to Northwestern University (AST 05-05230) and University of Chicago (AST 05-05124).

\section{REFERENCES}

Blake, G. A., Sandell, G., van Dishoeck, E. F., Groesbeck, T. D., Mundy, L. G., \& Aspin, C. 1995, ApJ, 441, 689

Chandrasekhar, S., \& Fermi, E. 1953, ApJ, 118, 113

Choi, M. 2001, ApJ, 553, 219

Choi, M. 2005, ApJ, 630, 976
Crutcher, R. M., Hakobian, N., \& Troland, T. H. 2009, ApJ, 692, 844

Di Francesco, J., Myers, P. C., Wilner, D. J., Ohashi, N., \& Mardones, D. 2001, ApJ, 562, 770 (DF2001)

Fiege, J. D., \& Pudritz, R. E. 2000, ApJ, 544, 830

Fielder, R. A., \& Mouschovias, T. C. 1993, ApJ, 415, 680

Galli, D., \& Shu, F. H. 1993, ApJ, 417, 220

Girart, J. M., Rao, R., \& Marrone, D. 2006, Science, 313, 812 (G2006)

Goncalves, J., Galli, D., \& Girart, J. M. 2008, A\&A, 490, L39

Goncalves, J., Galli, D., \& Walmsley, M. 2005, A\&A, 430, 979

Hatchell, J., Richer, J. S., Fuller, G. A., Qualtrough, C. J., Ladd, E. F., \& Chandler, C. J. 2005, A\&A, 440, 151

Hildebrand, R. H. 1983, Q. J. R. Astron. Soc., 24, 267

Hildebrand, R. H., Dotson, J. L., Dowell, C. D., Schleuning, D. A., \& Vaillancourt, J. E. 1999, ApJ, 516, 834

Hildebrand, R. H., Kirby, L., Dotson, J. L., Houde, M., \& Vaillancourt, J. E. 2009, ApJ, 696, 567

Houde, M., Vaillancourt, J. E., \& Hildebrand, R. H. 2009, in preparation

Lai, S., Crutcher, R. M., Girart, J. M., \& Rao, R. 2002, ApJ, 566, 925

Leung, C. M., \& Brown, R. L. 1977, ApJ, 214, L73

Li, H., Dowell, C. D., Kirby, L., Novak, G., \& Vaillancourt, J. E. 2008, Appl. Opt., 47, 422

Li, H., \& Houde, M. 2008, ApJ, 677, 1151

Machida, M. N., Matsumoto, T., Hanawa, T., \& Tomisaka, K. 2006, ApJ, 645, 1227

Matthews, B., McPhee, C., Fissel, L., \& Curran, R. 2009, ApJS, 182, 143

McKee, C. F., \& Ostriker, E. C. 2007, ARA\&A, 45, 565

Mouschovias, T. Ch., \& Spitzer, L., Jr. 1976, ApJ, 210, 326

Myers, P. C., Mardones, D., Tafalla, M., Williams, J. P., \& Wilner, D. J. 1996, ApJ, 465, L133

Nakamura, F., \& Li, Z. 2008, ApJ, 687, 354

Press, W. H., Teukolsky, S. A., Vetterling, W. T., \& Flannery, B. P. 2002, Numerical Recipes in C (2nd ed.; Cambridge: Cambridge Univ. Press)

Ostriker, E. C., Stone, J. M., \& Gammie, C. F. 2001, ApJ, 546, 980

Padoan, P., Goodman, A., Draine, B. T., Juvela, M., Nordlund, A., \& Rögnvaldsson, Ö. E. 2001, ApJ, 559, 1005

Rao, R., Crutcher, R. M., Plambeck, R. L., \& Wright, M. C. H. 1998, ApJ, 502, $75 \mathrm{~L}$

Sandell, G., Aspin, C., Duncan, W. D., Russell, A. P. G., \& Robson, E. I. 1991, ApJ, 376, L17

Shu, F., Adams, F., \& Lizano, S. 1987, ARA\&A, 25, 23

Tomisaka, K., Ikeuchi, S., \& Nakamura, T. 1988, ApJ, 326, 208

Vrba, F. J., Coyne, G. V., \& Tapia, S. 1993, AJ, 105, 1010 